\title{
COMORBIDITY AND SUICIDALITY IN PATIENTS DIAGNOSED WITH PANIC DISORDER/AGORAPHOBIA AND MAJOR DEPRESSION
}

\author{
Borjanka Batinic $^{1}$, Goran Opacic ${ }^{1}$, Tijana Ignjatov ${ }^{1}$ \& David S. Baldwin ${ }^{2}$ \\ ${ }^{1}$ Faculty of Philosophy, Belgrade, Serbia \\ ${ }^{2}$ University of Southampton, Southampton, United Kingdom
}

received: 28.9.2016;

revised: 15.2.2017;

accepted: 8.3.2017

\section{SUMMARY}

Background: Comorbidity of anxiety and depression (both current and lifetime) is associated with greater chronicity and an increased risk of suicidality. We wished to ascertain which symptom clusters had the strongest association with suicidality. Our aims were (1) to examine the presence of current comorbidity and suicidality in patients diagnosed with panic disorder/agoraphobia (PD/A) and major depression (MD), and their relationship with duration of psychiatric treatment and frequency of hospital admission; and (2) to examine which coexisting symptoms were most strongly predictive of suicidality in sub-groups and the overall group.

Subjects and methods: The study sample comprised 100 patients with PD/A and MD. The following assessment instruments were applied: the Panic and Agoraphobia Scale, the Beck Anxiety Inventory, the Beck Depression Inventory-II, the Beck Scale for Suicide Ideation, the Obsessive-Compulsive Inventory-Revised, the Liebowitz Social Anxiety Scale and the Whiteley Index of Hypochondriasis.

Results: High rates of current comorbidity were seen in both groups. Patients with MD had significantly higher suicidality scores, but were also older, with a longer duration of psychiatric treatment and more frequent hospitalizations. In the overall group, psychiatric comorbidity was correlated with duration of psychiatric treatment and frequency of hospitalizations (with the exception of hypochondriasis which was not correlated with frequency of hospitalization). In both sub-groups and the overall group, suicidality was correlated with scores for all examined comorbidity (with the exception of hypochondriasis in the PD/A group): however, after multiple regression only obsessive-compulsive symptomatology predicted suicidality in all sub-groups and the overall group, as well as depression in the overall group. Depression supposed as dependent variable and obsessive-compulsive symptomatology as a mediator explained around $37 \%$ of the variance in suicidal ideation.

Conclusion: Patients with PD/A or MD show high rates of current comorbidity. The effect of depression on suicidality was significant, but a non-trivial impact was also mediated by obsessive-compulsive symptomatology.

Key words: panic disorder/agoraphobia - major depression - comorbidity - suicidality

\section{INTRODUCTION}

Anxiety disorders and depressive disorders show considerable current and lifetime comorbidity in community and clinical settings. Lamers et al. (2011) found that in individuals with a current anxiety disorder, $63 \%$ had current and $81 \%$ lifetime comorbid depressive disorder, whereas in individuals with a current depressive disorder, $67 \%$ had current and $75 \%$ lifetime comorbid anxiety disorder: comorbidity of anxiety and depressive disorders being associated with higher prevalence of childhood trauma, earlier age of onset of the first disorder, longer duration of depressive and/or anxiety symptoms, and greater symptom severity. In primary care between $10-20 \%$ of adults in any given 12 months visit a physician during an anxiety or depressive disorder, more than $50 \%$ of these patients having secondary comorbid anxiety or depressive disorders (Hirschfield 2001). A prospective, 4-year community study found that the presence of any anxiety disorder, and each of the anxiety disorders (social phobia, agoraphobia, panic disorder, and generalized anxiety disorder), was associated with an increased risk of onset of major depressive disorder: this risk being greatest in the presence of more than one anxiety disorder, severe impairment due to anxiety disorder, and presence of panic attacks; severe psychosocial impairment being the strongest predictor of major depressive disorder (Bittner et al. 2004). Comorbidity of anxiety and depression is reflected in higher illness severity, greater chronicity, slower recovery, increased rates of recurrence, greater psycho-social impairment, more frequent use of medical resources, less satisfactory response to antidepressant treatment and higher risk of suicide (Hofmeijer-Sevink et al. 2012, Baldwin \& Lopes, 2009, De Graaf et al. 2004, Hirschfield 2001).

Although categorical systems of nosology consider anxiety and depression as distinct disorders, high rates of anxiety and depression comorbidity could be better explained by a dimensional approach to psychopathology. Epidemiological studies support both a categorical and dimensional conceptualization of anxiety and depression. The categorical concept holds that anxiety and depression are distinct disorders, with anxiety symptoms reflecting mental and physical activation, and depressive symptoms reflecting depletion (Mullaney 1984, Widlocher et al. 1983): although both disorders share a central theme of negative affect, depression is 
characterized by anhedonia (and low positive affect), whereas physiological hyperarousal is the central focus in anxiety disorders (Clark \& Watson 1991). By contrast, the dimensional view proposes that anxiety, depression and comorbid conditions lie on a continuum, and represent differing manifestations of the same underlying disorder: this conceptualization is supported by the symptomatic overlap of the two syndromes, the instability of clinical diagnosis, the tendency for patients suffering from long-lasting anxiety to develop depressive symptoms, the failure to find separate dimensions of anxiety and depression in self-rated and observer-rated scales, and the lack of differential responses to drug treatment (Linden 1998, Stavrakaki \& Vargo 1986). One unitary hypotheses highlights a pivotal role for 'neuroticism' - a stable personality trait defined by emotional instability as a key factor in vulnerability to stress and anxiety. Meta-analytic findings (Kotov et al. 2010) suggest that 'common mental disorders' (i.e. anxiety, depressive and substance use disorders) have similar trait profiles, with a common feature of neuroticism. High neuroticism characterises anxiety and depressive disorders in acute and remission phases, and is associated with the course of the disorder, co-morbidity and response to treatment (Batinic et al. 2013, Costa et al. 2005, Cuijpers et al. 2005, Bienvenu et al. 2004, 2001, Bienvenu \& Stein 2003, Widiger \& Trull 1992).

Anxiety disorders and depressive disorders are associated with an increased risk of suicide and non-fatal self-harm. A population-based longitudinal study in adults (Sareen et al. 2005) indicated that a pre-existing anxiety disorder is an independent risk factor for the subsequent onset of suicidal ideation and attempt. An epidemiological study of the relationship between anxiety disorders and suicide attempts found that panic disorder was significantly associated with lifetime suicide attempts, over and above the association with psychiatric co-morbidity (Nepon et al. 2010). However the association of panic disorder with suicidal behaviour may primarily explained by comorbidity with depressive disorders (Diaconu \& Turecki 2007), and the influence of comorbidity on mediating suicidality has not been studied extensively. A longitudinal study of risk factors for suicide attempts in major depressive disorder indicated that specific comorbid anxiety, personality, and substance use disorders was associated with incident suicide attempts (Bolton et al. 2008).

These findings suggest that co-morbidity confers an increased risk of future suicide attempts in patients with major depressive disorder, that is not solely accounted for by past suicidal behaviour. Unfortunately, in clinical practice co-morbidity is often under-recognized and under-treated (Jakovljevic \& Ostojic 2013). Our study represents an extension of previous studies of anxiety and depression comorbidity and its correlation to suicide risk. It extends consideration of this comorbidity in patients diagnosed with panic disorder/agoraphobia and major depression and its correlation with suicide risk, in order to determine the symptom cluster that most contributes to suicide across the two diagnoses. In their natural course, panic disorder/agoraphobia and major depression are frequently comorbid with each other, as well as with generalized anxiety disorder, social anxiety disorder, obsessive-compulsive disorder and hypochondriasis, which was our reason for including these comorbidities in the study.

Therefore, the aims of our study were (1) to examine the presence of current comorbid disorders and suicidality in patients diagnosed with panic disorder/agoraphobia (PD/A) and major depression (MD), and their correlation with the length of psychiatric treatment and frequency of psychiatric admission; and (2) to determine which of the comorbid symptoms were the most predictive for suicidality in $\mathrm{PD} / \mathrm{A}$ and $\mathrm{MD}$ sub-groups and the overall group (PD/A+MD).

According to previous, we hypothesized the following:

- H1: There would be a high rate of current comorbidity in both diagnostic groups.

- H2: In both groups there would be positive correlations between the presence of examined comorbidity and the duration of psychiatric treatment and the frequency of admission.

- H3: There would be a difference in mean scores on the suicidality scale between groups, patients with major depression having a higher score than patients with $\mathrm{PD} / \mathrm{A}$.

- H4: In both groups, and the overall group suicidality scores would correlate with scores of panic /agoraphobia, depression, generalized anxiety, social anxiety, obsessive-compulsive symptoms, and hypochondria.

\section{SUBJECTS AND METHODS}

\section{Study sample}

Participants were recruited among outpatients and inpatients who were receiving treatment at New Knezevac Hospital for Neuropsychiatric Disease between April 2011 and June 2012. The study sample recruited the patients which were clinically diagnosed by their treating psychiatrists according to ICD-10 (WHO 1992) criteria for current panic disorder with/without agoraphobia, or depressive disorder (single episode or recurrent, without psychotic features). Patients with bipolar depression were not included in the study. The Institutional Board approved the investigation and study participants provided written informed consent. Participants completed the study measures across two visits (outpatients) or during a single assessment session (inpatients).

\section{Assessment measures}

The following self-report assessments instruments were employed: Panic and Agoraphobia Scale (PAS) (Bandelow 1995), Beck Depression Inventory-II (BDIII) (Beck et al. 1996), Beck Scale for Suicidal Ideation 
(BSS) (Beck \& Steer 1993), Obsessive-Compulsive Inventory-Revised (OCI-R) (Foa et al. 2002), Liebowitz Social Anxiety Scale (LSAS) (Liebowitz 1987), Beck Anxiety Inventory (BAI) (Beck et al. 1998), and Whiteley Index of Hypochondriasis (WI) (Pilowsky 1967). Study participants also underwent a semistructured interview comprising questions relating to sociodemographic and clinical characteristics (including duration of psychiatric treatment determined by the time elapsed since first psychiatric encounter, and frequency of psychiatric admissions in the treatment period).

\section{Panic and Agoraphobia Scale}

The PAS estimates the severity of symptoms of panic disorder with or without agoraphobia. It comprises 13 items based on a five-point Likert scale (where $0=$ no symptoms, and $4=$ highest level of symptom severity) and contains 5 sub-scales measuring frequency of panic attacks, agoraphobia symptoms and avoidance behaviour, anticipatory anxiety, disability and fear for health concerns. The scale is widely used in clinical and research settings due its compatibility with DSM-IV and ICD-10 classifications. We used the self-rated version, which had high internal consistency $(\alpha=0.854)$.

\section{Beck Depression Inventory-II}

The BDI-II is used widely to assess depressive symptom severity in clinical samples and the general population, and comprises 21 items. Respondents evaluated how often in the last week they had experienced any of the symptoms on a four-point Likert scale (where $0=$ never, and $3=$ permanent). It showed high internal consistency $(\alpha=0.946)$. We have excluded item 9 of the scale (accessing suicidality).

\section{Beck Scale for Suicide Ideation}

The BSS comprises a 21-item scale preceded by five screening items, intended to assess thoughts, plans and intent to commit suicide. All 21 items are rated on a three-point scale (0 to 2 ), higher scores implying greater suicide risk. It had high internal consistency $(\alpha=0.847)$.

\section{Obsessive-Compulsive Inventory-Revised}

The OCI-R is used to determine the distress and severity of symptoms which characterize obsessivecompulsive disorder during the past month. It contains 18 items, each assessed on a five-point Likert scale (where $0=$ 'not at all distressed or bothered', and 4="extremely distressed or bothered'), and has six symptom domains (washing, checking, ordering, ruminating, neutralizing and hoarding). It has good testretest reliability and discriminated validity in clinical and non-clinical samples: it had high internal consistency $(\alpha=0.924)$.

\section{Liebowitz Social Anxiety Scale}

The LSAS assesses social anxiety symptoms and avoidance. It comprises 24 items divided into two main sub-scales (performance anxiety and avoidance of social situations). Each item is evaluated on a four-point Likert scale from 0 to 3 (corresponding to 'never', 'occasionally', 'often', and 'usually'). In our sample the LSAS had high internal consistency $(\alpha=0.977)$.

\section{Beck Anxiety Inventory}

Self-rating measure comprising 21 items to evaluate symptom severity. Respondents estimate how much they have been bothered by the symptoms during the last month on the scale of 0 ("not bothering me at all') to 3 ('severely, hardly bearable"). The BAI discriminates between anxious and non-anxious groups in different clinical populations. In our sample it showed high internal consistency $(\alpha=0.949)$.

\section{Whiteley Index of Hypochondriasis}

A self-report measure comprising 14 items (related to the fear of illness, beliefs about the existence of the disease, body preoccupations), each ranked on a fivepoint Likert scale (where 1='not at all true', and 5='it is quite true $\left.{ }^{6}\right)$. It had high internal consistency ( $\left.\alpha=0.914\right)$.

\section{Statistical analysis}

Analyses were conducted using Statistical Package for the Social Sciences (IBM SPSS Statistics 22). Internal consistency of study measures was determined by Cronbach's $\alpha$. Descriptive statistics were given separately for the two patient groups, using t-tests for independent samples, applying Bonferroni correction for all pair-wise comparisons. Applied instruments were standardised and normalised using Blooms method for refraction. Central tendencies of some data were reported using medians and standard deviations. Crosscorrelations between variables are given separately for each patient group. Multiple regression analysis was employed to examine the relationships between comorbidity and suicidality.

\section{RESULTS}

\section{Study sample}

The study sample comprised 100 patients - 50 diagnosed with panic disorder/agoraphobia and 50 with major depressive disorder without psychotic features. Women were in the majority in both groups (PD/A group, $72 \%$ women, $28 \%$ men; MD group, $66 \%$ women, $34 \%$ men). In the PD/A group, mean age was 36.12 $(\mathrm{SD}=8.886)$ years, and the mean length of psychiatric treatment was 41.36 ( $\mathrm{SD}=46.613)$ days: in the $\mathrm{MD}$ group, the mean age was $44.54(\mathrm{SD}=11.608)$ years, and the mean length of psychiatric treatment was 80.30 days 
$(\mathrm{SD}=85.933)$. The majority of subjects $(\mathrm{N}=68)$ had a high school education, followed by university education $(\mathrm{N}=73)$ and basic education $(\mathrm{N}=11)$.

\section{Associations with suicidality: overall sample}

Age was positively correlated with the level of suicidal ideation $(\mathrm{r}=0.267 ; \mathrm{p}<0.01)$ in the overall sample. In the overall sample, the frequency of hospitalizations was significantly and positively correlated with the level of suicidality, as was the length of psychiatric treatment, and all forms of comorbid symptoms (except for symptoms of hypochondriasis) even when age was controlled (see Table 1).

\section{Between-group comparisons: demographics}

The two groups differed significantly by age: MD patients having a significantly higher mean age (44.54 years; $\mathrm{SD}=11.608)$ when compared to patients with $\mathrm{PD} / \mathrm{A}(\mathrm{M}=36.12 ; \mathrm{SD}=8.886): \mathrm{F}=16.587 ; \mathrm{DF}=1 ; \mathrm{p}<0.01$. There were no significant differences between groups in gender distribution $\left(\chi^{2}=0.421 ; \mathrm{DF}=1 ; \mathrm{p}>0.05\right)$. There was a significant difference between groups in the length of psychiatric treatment between sub-samples: subjects diagnosed with MD had a significant longer mean duration of psychiatric treatment $(\mathrm{M}=80.30$ days, $\mathrm{SD}=85.933$ ) compared to patients diagnosed with $\mathrm{PD} / \mathrm{A}$ $(\mathrm{M}=41.36, \quad \mathrm{SD}=46.613) \quad(\mathrm{F}=7.933 ; \mathrm{DF}=1 ; \operatorname{sig}<0.01)$. Patients diagnosed with MD reported a significantly higher number of hospitalizations than patients diagnosed with $\mathrm{PD} / \mathrm{A}\left(\chi^{2}=23.874, \mathrm{DF}=7, \mathrm{p}<0.01\right)$.

\section{Between-group comparisons: comorbid syndromes}

There were significant differences between groups in mean scores on the Panic and Agoraphobia Scale, Beck Scale for Suicide Ideation and Beck Depression Inventory-II, with no significant differences between groups on the other measures (Table 2). As expected, patients diagnosed with $\mathrm{PD} / \mathrm{A}$ scored significantly higher on the Panic and Agoraphobia Scale (mean 24.32, $\mathrm{SD}=8.0444)$ as compared to patients diagnosed with MD (mean 16.0, $\mathrm{SD}=13.937)(\mathrm{p}<0.05)$ : by contrast, patients diagnosed with MD scored significantly higher on the Beck Depression Inventory-II (mean 29.26, $\mathrm{SD}=13.416)$ as compared to patients diagnosed with $\mathrm{PD} / \mathrm{A}$ (mean 16.04, $\mathrm{SD}=10.644, \mathrm{p}<0.05$ ). Patients diagnosed with MD scored significantly higher on the Beck Scale for Suicide Ideation (mean 5.70, $\mathrm{SD}=8.137$ versus mean $0.98, \mathrm{SD}=3.127, \mathrm{p}<0.05)$.

Table 1. Correlations and partial correlations of frequency of hospitalizations and length of treatment with applied instruments in the overall study sample

\begin{tabular}{|c|c|c|c|c|c|c|c|}
\hline \multicolumn{8}{|l|}{ Zero-order correlations } \\
\hline & BSS & PAS & BDI-II & BAI & L-SAS & OCI-R & WI \\
\hline Frequency of hospitalizations & $0.468 * *$ & $0.261 * *$ & $0.496^{* *}$ & $0.220 *$ & $0.464 * *$ & $444 * *$ & 0.169 \\
\hline Length of treatment & $0.441 * *$ & $0.228 *$ & $0.389 * *$ & $0.249 *$ & $0.443 * *$ & $0.388 * *$ & $0.234 *$ \\
\hline \multicolumn{8}{|c|}{ Partial correlations (age controlled) } \\
\hline & BSS & PAS & BDI-II & BAI & LSAS & OCI-R & WI \\
\hline Frequency of hospitalizations & $0.414 * *$ & $0.302 * *$ & $0.471 * *$ & $0.208 *$ & $0.463 * *$ & $0.416 * *$ & 0.180 \\
\hline Length of treatment & $0.374 * *$ & $0.282 * *$ & $0.352 * *$ & $0.240 *$ & $0.450 * *$ & $0.354 * *$ & $0.249 *$ \\
\hline
\end{tabular}

Table 2. Comorbid syndromes in the two groups

\begin{tabular}{lcrrrrr} 
& \multicolumn{3}{c}{ Panic/Agoraphobia } & \multicolumn{2}{c}{ Diagnosis } & \multicolumn{2}{c}{ Depression } & \multicolumn{1}{c}{ Total } \\
& \multicolumn{1}{c}{ M } & \multicolumn{1}{c}{ SD } & \multicolumn{1}{c}{ M } & SD & M & SD \\
\hline Panic and Agoraphobia Scale & $24.32_{\mathrm{a}}$ & 8.044 & $16.00_{\mathrm{b}}$ & 13.937 & 20.16 & 12.069 \\
Liebowitz Social Anxiety Scale & $99.90_{\mathrm{a}}$ & 31.649 & $112.36_{\mathrm{a}}$ & 36.733 & 106.13 & 34.682 \\
Obsessive-Compulsive Inventory-R & $19.08_{\mathrm{a}}$ & 13.900 & $23.52_{\mathrm{a}}$ & 14.929 & 21.30 & 14.523 \\
Beck Scale for Suicide Ideation & $0.98_{\mathrm{a}}$ & 3.127 & $5.70_{\mathrm{b}}$ & 8.137 & 3.34 & 6.575 \\
Beck Depression Inventory-II & $16.04_{\mathrm{a}}$ & 10.644 & $29.26_{\mathrm{b}}$ & 13.416 & 22.65 & 13.759 \\
Whiteley Index of Hypochondriasis & $43.84_{\mathrm{a}}$ & 11.458 & $39.40_{\mathrm{a}}$ & 13.082 & 41.62 & 12.436 \\
Beck Anxiety Inventory & $30.02_{\mathrm{a}}$ & 14.392 & $28.30_{\mathrm{a}}$ & 15.652 & 29.16 & 14.984 \\
\hline
\end{tabular}

Note: Values in the same row in sub-table not sharing the same subscript are significantly different at $\mathrm{p}<0.05$

in the two-sided test of equality for column means. The tests assume equal variances

${ }^{1}$ Tests are adjusted for all pairwise comparisons within a row of each innermost sub-table using the Bonferroni correction 
Table 3. Cross-correlations among variables ${ }^{1}$

\begin{tabular}{lcccccccc}
\hline & BSS & PAS & LSAS & OCI-R & BDI-II & WIH & BAI & PD/A (M) \\
\hline Beck Scale for Suicidal Ideation & & 0.344 & 0.234 & 0.456 & 0.298 & 0.197 & 0.304 & 0.306 \\
Panic and Agoraphobia Scale & 0.508 & & 0.427 & 0.586 & 0.582 & 0.601 & 0.593 & 0.522 \\
Liebowitz Social Anxiety Scale & 0.332 & 0.496 & & 0.612 & 0.49 & 0.378 & 0.446 & 0.431 \\
Obsessive-Compulsive Inventory-R & 0.632 & 0.583 & 0.649 & & 0.615 & 0.531 & 0.627 & 0.571 \\
Beck Depression Inventory-II & 0.590 & 0.704 & 0.702 & 0.734 & & 0.502 & 0.642 & 0.522 \\
Whiteley Index of Hypochondriasis & 0.407 & 0.709 & 0.639 & 0.618 & 0.654 & & 0.732 & 0.490 \\
Beck Anxiety Inventory & 0.379 & 0.702 & 0.533 & 0.543 & 0.609 & 0.789 & & 0.557 \\
\hline MD (M) & 0.475 & 0.617 & 0.559 & 0.627 & 0.666 & 0.636 & & \\
\hline PD/A+MD & BSS & PAS & LSAS & OCI-R & BDI & WIH & BAI & M-total \\
\hline Beck Scale for Suicidal Ideation & & & & & & & & 0.335 \\
Panic and Agoraphobia Scale & $0.236^{*}$ & & & & & & & 0.477 \\
Liebowitz Social Anxiety Scale & $0.337^{* *}$ & $0.377^{* *}$ & & & & & & 0.516 \\
Obsessive-Compulsive Inventory-R & $0.548^{* *}$ & $0.492^{* *}$ & $0.688^{* *}$ & & & & & 0.597 \\
Beck depression Inventory-II & $0.492^{* *}$ & $0.390^{* *}$ & $0.639^{* *}$ & $0.685^{* *}$ & & & & 0.529 \\
The Whiteley Index of Hypochondriasis & 0.176 & $0.693^{* *}$ & $0.531^{* *}$ & $0.577^{* *}$ & $0.445^{* *}$ & & & 0.536 \\
Beck Anxiety Inventory & $0.220^{*}$ & $0.673^{* *}$ & $0.521^{* *}$ & $0.593^{* *}$ & $0.522^{* *}$ & $0.791^{* *}$ & & 0.553 \\
\hline
\end{tabular}

M-average correlation; ** Significance at 0.01 level; * Significance at 0.05 level

${ }^{1}$ Upper main diagonal for $\mathrm{PD} / \mathrm{A}$, below upper diagonal for $\mathrm{MD}$

Table 4. Percentage of responders in both groups who met criteria for the other disorder

\begin{tabular}{|c|c|c|c|c|}
\hline & & . & & \\
\hline & & $\mathrm{PD} / \mathrm{A}$ & MD & Total \\
\hline Dg. Panic disorder/Agoraphobia & Count & 44 & 26 & 70 \\
\hline & $\%$ within & $62.9 \%$ & $37.1 \%$ & \\
\hline Dg. Social anxiety disorder & Count & 34 & 37 & 71 \\
\hline & $\%$ within & $47.9 \%$ & $52.1 \%$ & \\
\hline Dg. Obsessive-compulsive disorder & Count & 18 & 28 & 46 \\
\hline & $\%$ within & $39.1 \%$ & $60.9 \%$ & \\
\hline Dg. Major Depression & Count & 24 & 45 & 69 \\
\hline & $\%$ within & $34.8 \%$ & $65.2 \%$ & \\
\hline Dg Hypochondriasis & Count & 44 & 32 & 76 \\
\hline & $\%$ within & $57.9 \%$ & $42.1 \%$ & \\
\hline Dg .Generalized Anxiety disorder & Count & 29 & 26 & 55 \\
\hline & $\%$ within & $52.7 \%$ & $47.3 \%$ & \\
\hline
\end{tabular}

PD/A - Panic disorder/Agoraphobia; MD - Major Depression

\section{Cross-correlations between syndrome scores}

Scores on all applied instruments were standardised and normalised using Blooms method for refraction. Cross-correlations between variables are given separately for both groups, as well as for the overall group (PD/A+MD groups) in Table 3. Correlations between BSS and WIH were not statistically significant in all subgroups and in the overall group, but correlations between BSS and BDI, PD/A, LSAS, OCI-R and BAI were significant. In the $\mathrm{PD} / \mathrm{A}$ group, mean correlations were lower than in the MD sub-sample, and the Beck Scale for Suicide Ideation had the highest correlation with obsessive-compulsive symptomatology: in general terms, OCI-R scores had the highest correlations with all other variables in the $\mathrm{PD} / \mathrm{A}$ sample. In the MD group, OCI-R scores had the highest correlation with BSS, although the highest average correlation was with the BDI-II scale, which was the same in the overall $(\mathrm{PDA} / \mathrm{A}+\mathrm{MD})$ group.

\section{Syndromally-defined psychiatric comorbidity}

We explored syndromal comorbidity in both groups with reference to accepted 'cut-off 'scores. Where authors of the applied measures had suggested intervals for different levels of illness severity, we used the lower score value from the most severe category to classify the patient as meeting criteria for diagnosis (Table 4).

\section{Predictors of suicidal ideation}

Multiple regression analysis was employed to ascertain whether scores on particular measures were significant predictors of suicidal ideation, as measured by the Beck Scale for Suicide Ideation. Results are analyzed 
separately for each group and total sample as shown in Table 5. Identified predictors had better predictive power in the MD group $\left(\mathrm{R}_{\text {adjusted }}^{2}=0.414, \mathrm{p}=0.0\right)$ than in the PA group $\left(\mathrm{R}^{2}=234, \mathrm{p}=0.062\right)$. In the final analysis, obsessive compulsive symptoms remained as the only statistically significant predictor of suicidality through all groups, as well as for the overall group $(\mathrm{PD} / \mathrm{A}+$ $\mathrm{MD})$, as well as depression in the overall group.

\section{Obsessive-compulsive features and suicidality}

Given the predictive power of obsessive-compulsive symptoms, we undertook further analysis of individual OCI-R factors as predictors of suicidality through stepwise multiple regression in the whole sample. The items of the OCI-R scale which related to the factors of hoarding, mental neutralizing, ruminating, ordering and checking were the strongest predictors of suicidal ideation (Table 6).

As depression as well obsessive-compulsive symptomatology predicted suicidality in total sample, we employed additional statistics to explore relationships between suicidal ideation (BSS) on one side, depressive (BDI-II) and obsessive-compulsive (OCI-R) symptomatology deeper. Multiple mediation analysis SPSS macro (Preacher \& Hayes 2008) was applied. In the proposed partial mediation model BSS and BDI-II were supposed as dependent variables and OCI-R as a mediator. All other variables are considered as covariates. Direct and indirect effects of depressive symptoms are calculated. As it is shown in Table 7, total model explain around $37 \%$ of variance of suicidal ideation $\left(\mathrm{R}^{2}=0.3742, \mathrm{~F}(6\right.$, $93)=9.2687 \mathrm{p}<0.001)$. Covariates had no statistical significant impact on dependent variable.

Table 5. Predictors of sucidality

\begin{tabular}{lcccccc}
\hline & \multicolumn{3}{c}{ Standardized Coefficients } & \multicolumn{3}{c}{ Correlations } \\
PD/A & Beta & $\mathrm{T}$ & Sig. & Zero-order & Partial & Part \\
\hline Panic and Agoraphobia Scale & 0.175 & 0.926 & 0.360 & 0.344 & 0.140 & 0.124 \\
Liebowitz Social Anxiety Scale & -0.083 & -0.482 & 0.632 & 0.234 & -0.073 & -0.064 \\
Obsessive-Compulsive Inventory-R & 0.444 & 2.148 & 0.037 & 0.456 & 0.311 & 0.287 \\
Beck Depression Inventory-II & -0.007 & -0.038 & 0.970 & 0.298 & -0.006 & -0.005 \\
Whiteley Index of Hypochondriasis & -0.177 & -0.860 & 0.394 & 0.197 & -0.130 & -0.115 \\
Beck Anxiety Inventory & 0.093 & 0.408 & 0.685 & 0.304 & 0.062 & 0.054 \\
\hline MD & Beta & $\mathrm{T}$ & Sig. & Zero-order & Partial & Part \\
\hline Panic and Agoraphobia Scale & 0.160 & 0.868 & 0.390 & 0.508 & 0.131 & 0.095 \\
Liebowitz Social Anxiety Scale & -0.274 & -1.617 & 0.113 & 0.332 & -0.239 & -0.177 \\
Obsessive-Compulsive Inventory-R & 0.510 & 2.981 & 0.005 & 0.632 & 0.414 & 0.326 \\
Beck Depression Inventory-II & 0.350 & 1.720 & 0.093 & 0.590 & 0.254 & 0.188 \\
Whiteley Index of Hypochondriasis & -0.040 & -0.194 & 0.847 & 0.407 & -0.030 & -0.021 \\
Beck Anxiety Inventory & -0.044 & -0.234 & 0.816 & 0.379 & -0.036 & -0.026 \\
\hline PD/A+MD & Beta & $\mathrm{T}$ & Sig. & Zero-order & Partial & Part \\
\hline Panic and Agoraphobia Scale & 0.108 & 0.903 & 0.369 & 0.236 & 0.093 & 0.074 \\
Liebowitz Social Anxiety Scale & -0.100 & -0.816 & 0.416 & 0.337 & -0.084 & -0.067 \\
Obsessive- Compulsive Inventory-R & 0.554 & 4.133 & 0.000 & 0.548 & 0.394 & 0.339 \\
Beck Depression Inventory-II & 0.286 & 2.364 & 0.020 & 0.492 & 0.238 & 0.194 \\
Whiteley Index of Hypochondriasis & -0.193 & -1.297 & 0.198 & 0.176 & -0.133 & -0.106 \\
Beck Anxiety Inventory & -0.125 & -0.855 & 0.395 & 0.220 & -0.088 & -0.070 \\
\hline
\end{tabular}

Dependent variable: The Beck Suicidality Scale

Table 6. Changes in $\mathrm{R}^{2}$ after introducing each predictor of suicidality

\begin{tabular}{ccccccccc}
\hline & $\mathrm{R}$ & $\mathrm{R}^{2}$ & Adjusted $\mathrm{R}^{2}$ & \multicolumn{4}{c}{ Change Statistics } \\
& & & & $\mathrm{R}^{2}$ Change & F Change & df1 & df2 & Sig. F Change \\
\hline 1 & $0.534^{\mathrm{a}}$ & 0.285 & 0.278 & 0.285 & 39.127 & 1 & 98 & 0.000 \\
2 & $0.598^{\mathrm{b}}$ & 0.358 & 0.345 & 0.073 & 10.999 & 1 & 97 & 0.001 \\
3 & $0.639^{\mathrm{c}}$ & 0.408 & 0.389 & 0.050 & 8.049 & 1 & 96 & 0.006 \\
4 & $0.661^{\mathrm{d}}$ & 0.437 & 0.413 & 0.029 & 4.950 & 1 & 95 & 0.028 \\
5 & $0.686^{\mathrm{e}}$ & 0.471 & 0.443 & 0.034 & 5.990 & 1 & 94 & 0.016 \\
\hline
\end{tabular}

Predictors by steps:

1. I collect things I don't need; 2. I feel compelled to count while I am doing things; 3. I find it difficult to control my own thoughts;

4. I check things more often than necessary; 5. I need things to be arranged in a particular order 
Table 7. Direct and indirect effect of depression (BDI-II) on suicidality (BSS), with mediating effects of obsessivecompulsive symptomatology (OCI-R) on the whole sample

\begin{tabular}{lcccc}
\hline & Unstandardized Coefficient $^{*}$ & Standard error & $\mathrm{t}$ & $\mathrm{p}$ \\
\hline a - BDI-II to OCI-R & 0.3603 & 0.0910 & 3.9587 & 0.0001 \\
b - Direct Effects of OCI-R on BSS & 0.2506 & 0.0606 & 4.1331 & 0.0001 \\
c - Total Effect of BDI-II on BSS & 0.2269 & 0.0579 & 3.9195 & 0.0002 \\
c'- Direct Effect of BDI-II on BSS & 0.1367 & 0.0578 & 2.3645 & 0.0201 \\
axb - Indirect Effects of BDI-II & 0.0903 & 0.0420 & na $^{* *}$ & na \\
$\quad$ on BSS through OCI-R & & & & \\
\hline Partial Effect of Control Variables on BSS & Coefficient & se & $\mathrm{t}$ & $\mathrm{p}$ \\
\hline pas & 0.0589 & 0.0653 & 0.9029 & 0.3689 \\
lsas & -0.0190 & 0.0233 & -0.8165 & 0.4163 \\
wi & -0.1020 & 0.0786 & -1.2974 & 0.1977 \\
bai & -0.0550 & 0.0644 & -0.8550 & 0.3948 \\
\hline
\end{tabular}

${ }^{*}$ As result off all variables are normalized; $\quad{ }^{* *}$ Not applicable. Bootstrap method was used. $95 \%$ confidence interval after 1000 repeats did not contain 0 : that means that indirect effects is higher than 0

\section{DISCUSSION}

The study findings demonstrate the considerable level of current comorbidity seen in patients with $\mathrm{PD} / \mathrm{A}$ or MD, which confirmed our first hypothesis. These results concurs with previous studies of anxiety and depression comorbidity (Lamers et al. 2011, Bittner et al. 2004, Hirschfield 2001).

As for the hypothesis number two, the duration of psychiatric treatment from the first psychiatric encounter was positively correlated with scores on all examined comorbid syndromes in both subgroups, which accords with data on the positive association between longer duration of anxiety and depressive symptoms and comorbidity (Lamers et al. 2011). The frequency of hospitalizations during the period of treatment correlated positively with all the examined comorbid syndromes other than with hypochondriasis, which contrasts with the previous observation that patients with hypochondriasis with comorbid panic disorder had a higher trend towards health care utilisation (Hiller et al. 2005).

Our third hypothesis, namely that there would be a difference in mean scores on the suicidality scale between groups, and that the patients with MD would have a higher score than patients with $\mathrm{PD} / \mathrm{A}$, is also supported. Patients diagnosed with MD scored significantly higher on suicidality scores, which is in line with the data that major depression is the mental disorder the most related to suicidality (Berolote \& Fleichmann 2002). In both subgroups, measured suicidality was significantly positively correlated with age, length of psychiatric treatment, and frequency of hospitalizations in both groups, supporting previous findings (Trivedi et al. 2013, Qin \& Nordentoft 2005, Brown et al. 2000).

Our fourth hypothesis, that in both the sub-groups and the overall group, suicidality scores would correlate with scores of panic/agoraphobia, depression, generalized anxiety, social anxiety, obsessive-compulsive symptoms, and hypochondria, was supported with the exception of hypochondriasis in $\mathrm{PD} / \mathrm{A}$ subgroup: In both sub-groups and the overall group, suicidality was positively correlated with the level of panic/agoraphobia, depresson, generalized anxiety, social anxiety, and obsessive-compulsive symptoms. The lack of an association between hypochondriasis and suicidality in $\mathrm{PD} / \mathrm{A}$ patients is intriguing, as little is known about the relationships between hypochondriasis and suicidality. It seems possible that a preoccupation with fears of having serious medical illness and involvement in behaviours such as checking and reassurance-seeking may reduce suicidal thoughts. Our findings align with those from previous studies of suicidal behaviour in which panic disorder (and not simply through comorbidity with depression) was associated with lifetime suicide attempts (Nepon et al. 2010). Our findings support earlier observations that comorbidity amplifies the risk of suicidality (Bolton et al. 2008, Sareen et al. 2005). Panic disorder is also known to be an independent risk factor for suicide attempts in depressive illness (Katz et al. 2011). However, after multiple regression only obsessive-compulsive symptomatology significantly predicted suicidality in both sub-groups and the overall group, as well as depression in the overall group. Review of the literature on the relationship between suicidality in patients with obsessive-compulsive disorder found depression and hopelessness as a major correlates of suicidal behaviour (Kamath et al. 2007). Recent studies of the relationship between obsessivecompulsive symptoms and suicidality in students has shown that obsessive-compulsive symptomatology was not a significant risk factor for suicidality after controlling for depressive symptoms, although certain obsessive-compulsive symptom clusters, such as violent obsessions, can represent an independent risk factor for suicidality over and above depression (Huz et al. 2016, Ching et al. 2016). Our results emphasise the importance of detecting comorbid obsessive-compulsive symptoms in patients with panic disorder/agoraphobia and major depression. As patients often find it hard to describe obsessive-compulsive symptoms, mental health 
professionals need to ask specific questions about possible obsessive-compulsive cluster symptoms, particularly those relating to violent obsessions, and to hoarding, mental neutralizing, ruminating, ordering and checking.

As both depression and obsessive-compulsive symptomatology predicted suicidality in the overall group, an additional statistical analysis explored relationships between suicidal ideation, depression and obsessivecompulsive symptomatology and revealed that total effect of depression on suicidality was significant, but a non-trivial impact was also mediated by obsessivecompulsive symptomatology.

Further longitudinal research into coexisting symptoms and comorbid conditions in patients diagnosed with panic disorder/agoraphobia and major depression could clarify the nature of sequential comorbidity and influence of accumulating comorbidity on the level of suicidality.

\section{CONCLUSIONS}

Comorbidity of anxiety disorders and depression is the rule rather than the exception in clinical practice, and has important implications for prognosis, treatment decisions and risk of suicidality. As such, careful assessment for the presence of comorbid conditions should be an integral part of the clinical interview. Our findings suggest that early diagnosis and treatment of panic disorder/agoraphobia and major depression has the potential to reduce comorbidity, the length of psychiatric treatment and the frequency of hospital treatments, as well as the risk of suicidality. The study findings suggest that beside depression, coexisting obsessivecompulsive symptoms should be given particular consideration when examining the relationship between suicidality, panic disorder/agoraphobia and major depression, as well as in planning the most effective treatment interventions.

\section{Acknowledgements:}

The authors would like to thank the patients who participated in the study.

\section{Conflict of interest: None to declare.}

\section{Contribution of individual authors:}

Batinic Borjanka: design of the study, literature research, writing introduction, interpretation of the data, writing conclusions;

Opacic Goran: statystical analysis, interpretation of the data, study design;

Ignjatov Tijana: the sample collection, design of the study;

Baldwin S. David: mentorship of all the phases of the article development, supervision of English translation.

\section{References}

1. Baldwin DS \& Lopes TV: The influence of comorbid anxiety disorders on outcome in major depressive disorder. Medicographia 2009; 31:126-131.

2. Bandelow B: Assessing the efficacy of treatments for panic disorder and agoraphobia. II. The Panic and Agoraphobia Scale. International Clinical Psychopharmacology 1995; 10:73-82.

3. Batinic B, Saula-Marojevic B \& Vukosavljevic-Gvozden T: Personality profiles of patients with dysthymic and panic disorder. Psychiatria Danubina 2013; 25:115-122.

4. Beck A, Epstein N, Brown G \& Steer R: An inventory for measuring clinical anxiety: Psychometric properties. Journal of Consulting and Clinical Psychology 1998; 56:893-897.

5. Beck A, Steer $R$ \& Brown G: BDI-II, Beck depression inventory. San Antonio, Tex.: The Psychological Corporation, 1996.

6. Beck A \& Steer R: Beck scale for suicide ideation. San Antonio, Tex.: The Psychological Corporation, 1993.

7. Bertolote JM \& Fleischmann A: Suicide and psychiatric diagnosis: a worldwide perspective. World Psychiatry 2002; 1:181-185.

8. Bienvenu O, Samuels J, Costa P, Reti I, Eaton $W$ \& Nestadt G: Anxiety and depressive disorders and the fivefactor model of personality: A higher- and lower-order personality trait investigation in a community sample. Depression and Anxiety 2004; 20:92-97.

9. Bienvenu $O$, Brown $C$, Samuels J, Liang K, Costa P, Eaton $W$ et al.: Normal personality traits and comorbidity among phobic, panic and major depressive disorders. Psychiatry Research 2001; 102:73-85.

10. Bienvenu $O$ \& Stein M: Personality and Anxiety Disorders: A Review. Journal of Personality Disorders 2003; 17:139-151.

11. Bittner A, Goodwin RD, Wittchen HU, Beesdo K, Höfler M \& Lieb R: What characteristics of primary anxiety disorders predict subsequent major depressive disorder? The Journal of Clinical Psychiatry 2004; 65:618-26, quiz 730.

12. Bolton J, Cox B, Afifi T, Enns M, Bienvenu O \& Sareen J: Anxiety disorders and risk for suicide attempts: findings from the Baltimore Epidemiologic Catchment area followup study. Depression and Anxiety 2008; 25:477-481.

13. Brown $G$, Beck A, Steer $R$ \& Grisham J: Risk factors for suicide in psychiatric outpatients: A 20-year prospective study. Journal of Consulting and Clinical Psychology 2000; 68:371-377.

14. Ching TH, Williams $M$ \& Siev J: Violent obsessions are associated with suicidality in an OCD analog sample of college students. Cogn Behav Ther 2016; 23:1-12.

15. Clark L \& Watson D: Tripartite model of anxiety and depression: Psychometric evidence and taxonomic implications. Journal of Abnormal Psychology 1991; 100:316-336.

16. Costa P, Bagby R, Herbst J \& McCrae R: Personality selfreports are concurrently reliable and valid during acute depressive episodes. Journal of Affective Disorders 2005; 89:45-55.

17. Cuijpers $P$, van Straten A \& Donker M: Personality traits of patients with mood and anxiety disorders. Psychiatry Research 2005; 133:229-237.

18. De Graaf R, Bijl R, ten Have M, Bekman A \& Vollebergh, W: Pathways to comorbidity: the transition of pure mood, anxiety and substance use disorders into comorbid 
conditions in a longitudinal population-based study. Journal of Affective Disorders 2004; 82:461-467.

19. Diaconu $G$ \& Turecki G: Panic disorder and suicidality: Is comorbidity with depression the key? Journal of Affective Disorders 2007; 104:203-209.

20. Foa EB, Huppert JD, Leiberg S, Langner R, Kichic R, Hajcak $G$ et al.: Obsessive-Compulsive Inventory: Development and validation of a short version. Psychological Assessment 2002; 14:485-496.

21. Hiller W, Leibbrand R, Rief W \& Fichter M: Differentiating hypochondriasis from panic disorder. Journal of Anxiety Disorders 2005; 19:29-49.

22. Hirschfield R: The Comorbidity of Major Depression and Anxiety Disorders. The Primary Care Companion to The Journal of Clinical Psychiatry 2001; 03:244-254.

23. Hofmeijer-Sevink M, Batelaan N, van Megen H, Penninx $B$, Cath $D$, van den Hout $M$ et al.: Clinical relevance of comorbidity in anxiety disorders: A report from the Netherlands Study of Depression and Anxiety (NESDA). Journal of Affective Disorders 2012; 137:106-112.

24. Huz I, Nyer M, Dickson C, Farabaugh A, Alpert J, Fava M \& Baer L: Obsessive-Compulsive Symptoms as a Risk Factor for Suicidality in U.S. College Students. J Adolesc Health 2016; 58:481-4.

25. Jakovljevic M \& Ostojic L: Comorbidity and multimorbidity in medicine today: challenges for bringing separated branches of medicine closer to each other. Psychiatria Danubina 2013; 25:18-28.

26. Kamath P, Reddy YC \& Kandavel T: Suicidal behavior in obsessive-compulsive disorder. J Clin Psychiatry 2007; 68:1741-50.

27. Katz C, Yaseen Z, Mojtabai R, Cohen L \& Galynker I: Panic as an Independent Risk Factor for Suicide Attempt in Depressive Illness. The Journal of Clinical Psychiatry 2011; 72:1628-1635.

28. Kotov R, Gamez W, Schmidt F \& Watson D: Linking "big" personality traits to anxiety, depressive, and substance use disorders: A meta-analysis. Psychological Bulletin 2010; 136:768-821.

29. Lamers F, van Oppen P, Comijs H, Smit J, Spinhoven $P$, van Balkom A et al.: Comorbidity Patterns of Anxiety and Depressive Disorders in a Large Cohort Study (NESDA). The Journal of Clinical Psychiatry 2011; 72:341-348.
30. Liebowitz MR: Social phobia. Modern Problems in Pharmacopsychiatry 1987; 22:141-173.

31. Linden M: The clinical interrelation between anxiety and mood disorder. Presented at the 11th ECNP Congress, 31, October-4 November 1998. Paris, France.

32. Mullaney J: The relationship between anxiety and depression. Journal off Affective Disorders 1984; 7:139148.

33. Nepon J, Belik S, Bolton J \& Sareen J: The relationship between anxiety disorders and suicide attempts: findings from the National Epidemiologic Survey on Alcohol and Related Conditions. Depression and Anxiety 2010; 27:791-798.

34. Pilowsky I: Dimensions of Hypochondriasis. The British Journal of Psychiatry 1967; 113:89-93.

35. Preacher KJ \& Hayes AF: Asymptotic and resampling strategies for assessing and comparing indirect effects in multiple mediator models. Behavior Research Methods 2008; 40:879-891.

36. Qin $P$ \& Nordentoft $M$ : Suicide Risk in Relation to Psychiatric Hospitalization. Archives Of General Psychiatry 2005; 62:427.

37. Sareen J, Cox B, Afifi T, de Graaf R, Asmundson G, ten Have $M$ et al.: Anxiety Disorders and Risk for Suicidal Ideation and Suicide Attempts. Archives of General Psychiatry 2005; 62:1249.

38. Stavrakaki C \& Vargo B: The relationship of anxiety and depression: a review of the literature. The British Journal of Psychiatry 1986; 149:7-16.

39. Trivedi $M H$, Morris DW, Wisniewski SR, Nierenberg AA, Gaynes BN, Kurian BT et al.: Clinical and sociodemographic characteristics associated with suicidal ideation in depressed outpatients. Canadian Journal of Psychiatry 2013; 58:113-22.

40. Widiger T \& Trull T: Personality and Psychopathology: An Application of the Five-Factor Model. Journal of Personality 1992; 60:363-393.

41. Widlocher D, Lecrubier $Y$ \& Le Goc Y: The place of anxiety in depressive symptomatology. British Journal of Clinical Pharmacology 1983; 15:172-179.

42. World Health Organization: The ICD-10 Classification of Mental and Behavioral Disorders. Geneva, 1992.

Correspondence:

Associate Professor Borianka Batinic, $M D, P h D$

Faculty of Philosophy, Department of Psychology, University of Belgrade

Clinic of Psychiatry, Clinical Center of Serbia

Pasterova 2, 11000, Belqrade, Serbia

E-mail:doubleb@eunet.rs 